\title{
Estudio teórico experimental sobre el fenómeno de enfriamiento postsoldadura en una unión soldada cruciforme
}

\author{
Theoretical and experimental study of the phenomenon of \\ post weld cooling in a cruciform welded joint \\ Oscar Javier Araque de los Ríos ${ }^{1} \quad$ Nelson Arzola de la Peña ${ }^{2}$ \\ Recibido 11 de febrero de 2015, aceptado 9 de septiembre de 2015 \\ Received: February 11, 2015 Accepted: September 9, 2015
}

\begin{abstract}
RESUMEN
En este trabajo se presenta un estudio sobre el comportamiento del campo de temperatura y del perfil de esfuerzos residuales que aparecen en una unión soldada tipo cruciforme durante la fase de enfriamiento postsoldadura. Se utiliza un modelo 3D y un análisis de transferencia de calor transciente no lineal mediante el método de los elementos finitos. La corroboración de las simulaciones obtenidas se logra experimentalmente construyendo probetas soldadas con geometría cruciforme utilizando el proceso SMAW, empleando como metal base placas en acero estructural ASTM HR A36 y metal de aporte correspondiente a electrodos E6013. Las probetas son sometidas a tres condiciones de enfriamiento: en agua, en aceite, y en aire. Se establece un campo de temperatura inicial definido de acuerdo con los datos termográficos obtenidos por vía experimental en el momento de la construcción de los testigos no estandarizados, los cuales se fabrican con tres tamaños distintos de cateto de soldadura y con un espesor de placa constante. Se encuentra muy buena correspondencia entre las curvas de enfriamiento experimentales y las curvas de enfriamiento de las simulaciones numéricas. Como conclusiones fundamentales se obtiene que tanto el tamaño de la región afectada por los esfuerzos residuales como las magnitudes pico de estos están influenciadas por el medio de enfriamiento; medios de enfriamiento que provocan tasas de enfriamiento altas, generan regiones de esfuerzos residuales, cuyas magnitudes son más altas. Además, se observó un incremento de los esfuerzos residuales para una mayor dimensión del cateto de soldadura.
\end{abstract}

Palabras clave: Unión soldada cruciforme, tasa de enfriamiento, esfuerzo residual, perfil de temperatura.

\section{ABSTRACT}

This paper presents a study on the behavior of the temperature field and the profile of residual stresses that appear in a type cruciform welded joint during post weld cooling phase. A 3D model and an analysis of nonlinear transient heat transfer by the finite elements are used. Corroboration of the simulations were done experimentally, achieved by constructing welded cruciform specimen geometry using the SMAW process, using as a base contribution metal plates HR ASTM A36 structural steel and metal electrodes corresponding to E6013 contribution. Specimens were subjected to three cooling conditions: water, oil, and air. A field defined initial temperature according to thermo-graphics data that were obtained experimentally during the construction of not standardized specimens, which were made from three different hick welding sizes, while plate thickness where kept constant. Very good correspondence between both cooling curves obtained experimentally and by numerical simulations was found. This work shows that the size of the region affected by residual stresses and peak magnitudes are influenced by the cooling medium. High cooling medium produce high cooling rates, generating residual stresses regions of bigger magnitudes. Furthermore, an increase in the residual stress along the welding hick was observed.

Keywords: Cruciform welded joint, cooling rate, residual stress, temperature profile.

1 Grupo de Investigación Desarrollo Tecnológico. Departamento de Ingeniería Mecánica. Universidad de Ibagué, Colombia. E-mail: oscar.araque@unibague.edu.co

2 Grupo de Investigación en Diseño Óptimo Multidisciplinario. Departamento de Ingeniería Mecánica y Mecatrónica. Universidad Nacional de Colombia. Bogotá, Colombia. E-mail: narzola@unal.edu.co 


\section{INTRODUCCIÓN}

El estudio de los procesos de soldadura presenta dificultades muy específicas, sobre todo por la naturaleza transitoria y no lineal, al movimiento de la fuente de calor y a la dependencia de las propiedades del material con la temperatura [1]. Attarha y Far [2], han estudiado la distribución de la temperatura de juntas soldadas a tope en la zona afectada por el calor (ZAC) utilizando termocuplas tipo K; los datos experimentales obtenidos fueron comparados mediante simulaciones de modelos 3D, empleando el método de los elementos finitos (MEF) con ayuda del software ABAQUS. Los resultados experimentales y de simulación permiten predecir la distribución de la temperatura en las placas soldadas mediante el proceso de soldadura con electrodo de tungsteno y gas de protección inerte (TIG). En este estudio se encontró que existe un comportamiento no lineal, verificándose un alto grado de correlación entre los resultados experimentales y la simulación desarrollada mediante el MEF. Por otro lado, es posible predecir la microestructura de la zona afectada por el calor (ZAC) en función del cambio de temperatura. Rodríguez [3] relaciona las distintas zonas que conforman la estructura de una unión soldada de un acero de bajo carbono con las temperaturas alcanzadas mediante el diagrama $\mathrm{Fe}$ - C. La ZAC está dividida por varias subzonas, en función de las características estructurales y las propiedades del material que la forman. De acuerdo con la investigación, para las zonas situadas a continuación de la zona de normalizado (zona de recristalización incompleta, zona de recristalización y zona de fragilidad en azul) es difícil establecer la ubicación de sus bordes. Se considera además que el límite de la ZAC se encuentra en la zona de normalizado (conocida también como de grano fino) que se encuentra ligeramente por encima de la zona correspondiente al fin de la transformación de la perlita en austenita durante el calentamiento del acero, el investigador establece una expresión en función de la composición química para la determinación de la posición de esta zona. Otros investigadores han dirigido los estudios hacia el análisis del efecto de la temperatura máxima, la tasa de enfriamiento y el método de soldadura empleado. Lee y Wu [4] han determinado que la velocidad de enfriamiento de las uniones soldadas mediante el proceso de Haz laser es de alrededor de 212,6 ${ }^{\circ} \mathrm{C} / \mathrm{s}$, mientras que la velocidad de enfriamiento de uniones soldadas mediante el proceso TIG es de $17-20,6^{\circ} \mathrm{C} / \mathrm{s}$. El análisis metalográfico de la unión es coincidente con el comportamiento propuesto por Rodríguez [3]. Fratini [5] utilizó agua en el proceso de enfriamiento con el fin mejorar la calidad final de las juntas soldadas a tope en términos de resistencia mecánica. Como resultado obtuvo que en general el agua no posee un efecto perjudicial para la unión soldada. Por su parte, Hwa [6] estudia el efecto de la temperatura en uniones soldadas por el proceso TIG, encontrando una alta correlación entre las simulaciones numéricas y las pruebas experimentales. Shi y colaboradores [7] estudian la evolución de la estructura de grano en la ZAC, para un acero de grano fino SS400. Para ello se integraron simulaciones en 3-D de Monte Carlo (MC), para el crecimiento de grano de la ZAC de soldadura. El método simula la dinámica del proceso no isotérmico del crecimiento del grano en la ZAC de soldadura. Los tamaños de grano de la zona de grano grueso afectada por el calor (GGZAC) obtenidos de la simulación MC corresponden con la medición experimental de los cordones reales soldados. Además, la simulación indica que el grado de crecimiento de grano es más alto para el acero SS400 de grano ultrafino en comparación con el acero convencional. Con el aumento en la entrada de calor, el crecimiento de grano de la GGZAC aumenta rápidamente. Debido a que la energía de activación para el crecimiento del grano es menor para el acero de grano extremadamente fino (SS400), que para granos de austenita en acero convencional, estos pueden crecer en un nivel relativamente de temperatura más baja, por este motivo el intervalo de la GGZAC se ensancha. Perret [8] ha empleado métodos analíticos y numéricos para determinar el campo de temperatura debido a los efectos del calor de soldadura. En su trabajo concluye que es necesario tener en cuenta la dimensión finita y el tiempo en que ocurre el enfriamiento. Wahab y otros [9] determinan que por medio de la predicción del ciclo térmico es posible proporcionar una estimación de la profundidad de penetración de la soldadura, la geometría del charco y las velocidades de enfriamiento. Los tiempos de enfriamiento, la penetración de soldadura y la longitud de los charcos de soldadura se comparan con los valores obtenidos utilizando simulaciones de elementos finitos en 2D y 3D. Cuando se comparan los resultados teóricos con los experimentales de la unión real se aprecia un buen nivel de correlación. En la investigación 
de Shixiong, Jianling, Haitao y Shiqin [10] se utiliza la simulación de uniones soldadas mediante el proceso TIG para unir una aleación de cobre Hs201 sobre acero $35 \mathrm{CrMnSiA}$, estudiándose el comportamiento del flujo y el charco de fusión. Los resultados muestran que el ciclo térmico cambia notablemente, la temperatura máxima y la velocidad de enfriamiento en la interfaz de fusión es mayor que en las zonas alejadas de la interfaz. Los esfuerzos residuales pueden ser considerados como fuerzas internas que se mantienen actuando en un material después de manufacturado o procesado en ausencia de fuerzas externas o gradiente térmico. Los esfuerzos residuales se crean durante la mayoría de los procesos de manufactura que incluyen deformaciones del material, tratamiento térmico, maquinado u operaciones que producen cambios de forma o que cambian las propiedades del material [11]. Existen tres clases de esfuerzos residuales de acuerdo con la distancia o rango sobre el cual son analizados [12]; los denominados de tipo macroscópicos, que son de naturaleza de largo alcance y se extienden sobre varios granos del material; los de tipo microesfuerzos estructurales, que abarcan la distancia de un grano o parte de uno y pueden presentarse entre fases diferentes y tener características físicas distintas; y por último los esfuerzos residuales de tipo microscópico, que actúan sobre varias distancias interatómicas dentro de un grano. Para el caso de los procesos de soldadura, los esfuerzos residuales son producto de la interacción de los siguientes procesos separados [13]: a) expansión impedida y contracción resultante de una distribución de temperatura no homogénea, b) efectos de enfriamiento rápido (temple) y, c) transformaciones de fase. Consecuentemente, el tipo y cantidad de esfuerzos residuales en el cordón de soldadura y ZAC dependen de la temperatura de transformación, determinada por el diagrama tiempo-temperatura-transformación del material y la velocidad de enfriamiento; adicionalmente, la composición química local juega un papel importante [14]. Diversos métodos son utilizados para la determinación de la magnitud de los esfuerzos residuales, entre ellos pueden mencionarse la técnica del hoyo ciego, de la curvatura y el de difracción de rayos X [15]. Para la difracción de rayos X el procedimiento más utilizado para determinar los esfuerzos residuales es el "método de $\sin 2 \psi$ "; el que permite determinar la distribución de la deformación de la red cristalina en una fina capa superficial. La principal ventaja de este método es el establecimiento de la linealidad de la distancia interplanar en función de $\sin 2 \psi[16]$.

El desarrollo de la presente investigación pretende estudiar el comportamiento de una unión soldada, a solape en probetas de tipo cruciforme, para condiciones de enfriamiento en distintos medios (agua, aceite y aire) y empleando catetos de soldadura de varios tamaños ( $3 \mathrm{~mm}, 4 \mathrm{~mm}$ y $5 \mathrm{~mm}$ ), con el fin de determinar la influencia de estos factores en la magnitud de los esfuerzos residuales que se generan debido a las transformaciones metalúrgicas que se suceden y a las contracciones térmicas propias del proceso.

La relevancia de este trabajo radica en que estudia, desde perspectivas tanto teóricas como experimentales, la influencia que poseen el ciclo térmico postsoldadura y la geometría de la sección del cordón de soldadura en los esfuerzos residuales que surgen en las zonas fundidas y afectadas por el calor. La determinación de los esfuerzos residuales posibilita contar con modelos más acertados de predicción de falla estructural; lo anterior repercute en una gestión de vida más eficiente de las estructuras y los componentes soldados. Por ejemplo, al utilizar la Mecánica de la Fractura como herramienta de gestión de vida es posible determinar con mayor exactitud la tasa de propagación de grieta y el tamaño crítico de grieta, si se incorpora en el análisis el campo de esfuerzos residuales que es hallado por medio del procedimiento desarrollado en la presente investigación.

\section{MATERIALES Y MÉTODOS}

Con el propósito de justificar la utilización de la unión soldada cruciforme en el presente trabajo, se pudo identificar que esta configuración es una de las más empleadas como solución de unión estructural. Además, la geometría cruciforme posibilita la inspección y determinación del comportamiento para cuatro juntas soldadas simultáneamente, desde el punto de vista de su geometría, propiedades térmicas, mecánicas, defectos y resistencia. Por otro lado, se analizaron distintos tipos de tecnologías para la obtención de la unión, constatándose la amplia gama de tecnologías de unión mediante soldadura. Ente ellas se destacan: soldadura ordinaria o de aleación, soldadura por gas, soldadura por arco, soldadura por 
arco con electrodo recubierto, soldadura por arco con fundente en polvo, soldadura aluminotérmica, soldadura por presión, soldadura por resistencia, soldadura por ultrasonido, entre otras. Entre todas ellas, la soldadura manual con electrodo revestido (SMAW) es una de las más ampliamente usadas en el montaje estructural [17]. Por ello, en el presente trabajo se estableció el estudio de este tipo de soldadura, empleando como material base acero estructural ASTM HR A36 y como material de aporte electrodos E6013.

\section{Geometría y características de la probeta}

Para el desarrollo experimental se utilizó una chapa de acero al carbono ASTM HR A36 comercial, de $8 \mathrm{~mm}$ de espesor cuya composición química corresponde a la presentada en la Tabla 1.

El proceso de soldadura de las probetas cruciformes se realizó en las instalaciones de la empresa WEST ARCO, con personal calificado y empleando un equipo de soldadura marca Miller XMT $350 \mathrm{CC} /$ CV. Se llevaron a cabo 2 depósitos de filete, con junta en $\mathrm{T}$ soldado por ambos lados con bisel cuadrado, para cada probeta de una serie total de 50. Las características eléctricas del proceso SMAW son mostradas en la Tabla 2, para cada tamaño de cateto de soldadura.

La geometría y dimensiones de la probeta se presentan en la Figura 1. Para el proceso de corte de las placas se utiliza la técnica de plasma de alta densidad. La zona de afectación térmica para esta técnica está

Tabla 1. Composición química del acero ASTM HR A36 empleado (Fuente: Compañía Nacional de Aceros, código material 15833).

\begin{tabular}{|c|c|c|c|c|c|c|c|c|}
\hline $\mathbf{F e}$ & $\mathbf{C}$ & $\mathbf{S i}$ & $\mathbf{M n}$ & $\mathbf{S}$ & $\mathbf{P}$ & $\mathbf{N i}$ & $\mathbf{C r}$ & $\mathbf{N}$ \\
\hline 98,523 & 0,16 & 0,19 & 0,88 & 0,09 & 0,010 & 0,025 & 0,03 & 0,066 \\
\hline
\end{tabular}

Tabla 2. Especificación de los procedimientos de soldadura utilizados.

\begin{tabular}{|c|c|c|c|}
\hline $\begin{array}{c}\text { Cateto de } \\
\text { soldadura }\end{array}$ & $\begin{array}{c}\text { Diámetro } \\
\text { del } \\
\text { electrodo }\end{array}$ & $\begin{array}{c}\text { Parámetros } \\
\text { eléctricos } \\
\text { (V, A) - CD }\end{array}$ & $\begin{array}{c}\text { Velocidad } \\
\text { de avance } \\
\text { (mm/min) }\end{array}$ \\
\hline $3 \mathrm{~mm}$ & $3 / 32 "$ & $72 ; 102$ & 20 \\
\hline $4 \mathrm{~mm}$ & $1 / 8 ”$ & $69 ; 92$ & 20 \\
\hline $5 \mathrm{~mm}$ & $1 / 8 ”$ & 71,$2 ; 98$ & 20 \\
\hline
\end{tabular}

en el rango de 1 a $5 \mathrm{~mm}$. Para el caso del espesor de lámina de $8 \mathrm{~mm}$, la misma solo puede alcanzar como máximo un milímetro de profundidad respecto de la superficie que resulta del corte, lo que puede variar la microestructura superficial y la dureza. El acabado superficial que se logra se encuentra en el rango de 1 a $10 \mu \mathrm{m}$.

El diámetro de la boquilla utilizada para el equipo de corte es de 0,8 milímetros, las tolerancias para el corte en la pieza son de $(-0,23+0,08)$; para ello se debe considerar en el plano de corte con una tolerancia del agujero de la boquilla, por lo que se toma un espacio entre piezas de $1 \mathrm{~mm}$.

Con el propósito de establecer las tasas de enfriamiento para cada tamaño de cateto se somete la probeta a diversos medios de enfriamiento (ME), los cuales son establecidos como aparece en la Tabla 3. También se muestra el tiempo de permanencia para cada probeta en el medio de enfriamiento.

\section{Montaje experimental para el ensayo de enfriamiento} La medición de la temperatura de la probeta durante su enfriamiento se realizó con la ayuda de dos termocuplas tipo K, ubicadas lateralmente en cada
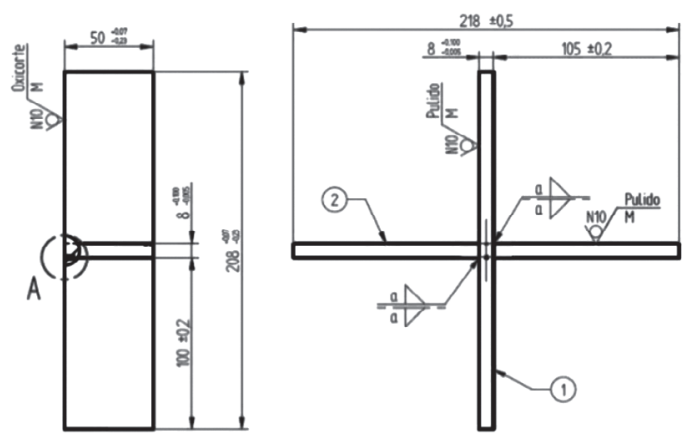

Figura 1. Geometría de la probeta cruciforme (medidas en $\mathrm{mm}$ ).

Tabla 3. Especificación acerca de los medios de enfriamiento utilizados.

\begin{tabular}{|l|c|c|}
\hline $\begin{array}{c}\text { Medio de } \\
\text { enfriamiento }\end{array}$ & $\begin{array}{c}\text { Tiempo de } \\
\text { permanencia } \\
\text { promedio } \\
(\mathbf{m i n})\end{array}$ & $\begin{array}{c}\text { Temperatura } \\
\text { ambiente }\left({ }^{\circ} \mathbf{C}\right)\end{array}$ \\
\hline Severo - Agua & 4 & 21,9 \\
\hline Intermedio - Aceite & 5 & 25,3 \\
\hline Leve - Aire & 90 & 26,7 \\
\hline
\end{tabular}


borde de donde se ejecuta el cordón de soldadura. Las termocuplas van conectadas a una tarjeta de adquisición de datos NI-9211, montada sobre el chasis NI-CDAQ-9172 y esta a su vez acoplada al $\mathrm{PC}$, como aparece en el diagrama presentado en la Figura 2. Para la visualización de los resultados se emplea el software LabView Signal Express 2011.

\section{Medición termográfica del campo de temperatura}

Se utiliza una cámara termográfica modelo Fluke Ti27 con el propósito de determinar el campo de temperatura en la superficie de las probetas y su evolución durante el proceso de enfriamiento pos soldadura. Esta información es de particular interés al momento de fidelizar los resultados de las simulaciones. En la Figura 3 se muestra una de

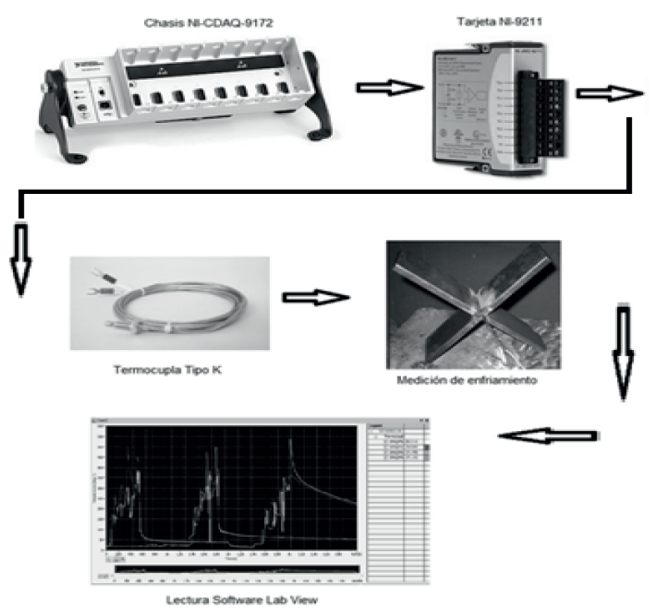

Figura 2. Esquema de conexión de los componentes de medición de la temperatura.

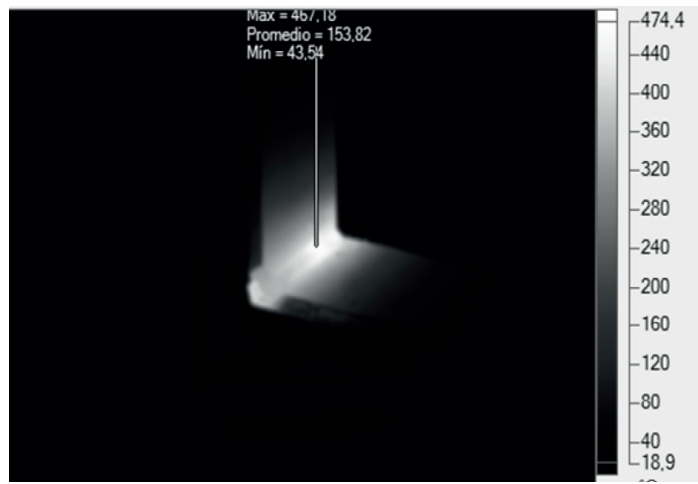

Figura 3. Imagen termográfica para la probeta con cateto de soldadura de $3 \mathrm{~mm}$. las imágenes que es posible obtener mediante el software SmartView 3.3.

\section{Perfil de ondulación superficial}

Para la construcción de un modelo tridimensional, que replique de manera fiel las condiciones geométricas de las superficies de las uniones soldadas, se determina el perfil de ondulación superficial utilizando un rugosímetro Mitutoyo serie SJ-210, como se muestra en la Figura 4 (a). Posteriormente, los valores obtenidos en el estudio se importan a un programa tipo CAD (SolidWorks Educational, Versión 2010-2011) para generar una curva similar a la superficie del cordón de soldadura. Luego, utilizando una foto ampliada del cordón se logra determinar una aproximación a la convexidad del cordón y a la curva real de los surcos que se generan durante la aplicación de la soldadura, como se muestra en la Figura 4 (b).

\section{Modelo para la probeta y condiciones de simulación}

El desarrollo de las simulaciones se realiza utilizando el software ANSYS 14.5 y APDL, debido a la funcionalidad y la capacidad que tiene este para el desarrollo del MEF. La elaboración del modelo CAD de las probetas es la tarea inicial de la formulación del modelo teórico, ya que de esta depende en gran medida los resultados de la simulación. El

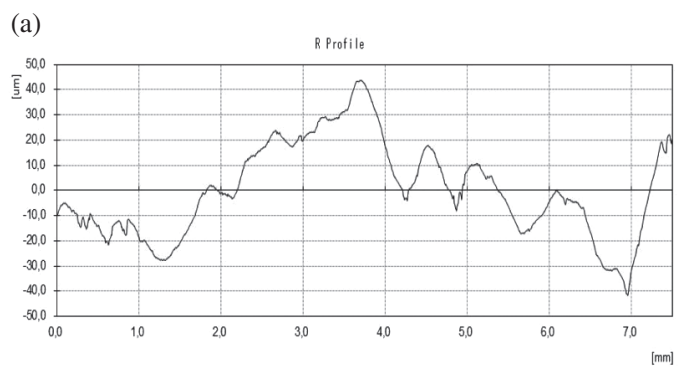

(b)

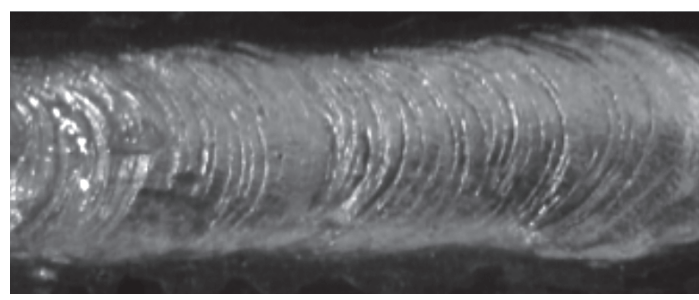

Figura 4. (a) Perfil de rugosidad medido para una de las probetas (AC2); (b) Fotografía superior del cordón de soldadura para una de las probetas. 
modelo CAD se realizó en el software SolidWorks 2010 en su versión académica, aprovechando la completa compatibilidad con el software ANSYS 14.5. Se emplea la interfaz de estudios Ansys Workbench 15.0, donde se ingresan los materiales (propiedades del material base, electrodo y ZAC) y tipo de análisis. Luego se procede a importar la geometría respectiva desde el software CAD. Las propiedades termofísicas de los materiales tales como conductividad térmica, densidad y calor específico se definen para este análisis térmico transitorio. Inicialmente se toma una malla generada automáticamente, y con posterioridad se realiza un refinamiento de la misma en las zonas de interés (bordes y pie de la soldadura), donde se debe enfocar el análisis con el fin de mejorar la calidad de los resultados. Se pudo determinar que entre 1,2 y 1,7 millones de nodos, según un análisis de convergencia de datos realizado, los resultados del sistema tienen un comportamiento similar, razón por la que se trabajó con una malla final con aproximadamente 1,5 millones de nodos. En la Figura 5 se observa un modelo de la probeta cruciforme, esta presenta una malla con refinamiento nivel 3 y tamaño de nodo de $0,001 \mathrm{~m}$ (para 1.540 .451 nodos).

Para la realización de las simulaciones se tuvieron en cuenta los controles básicos que se describen a continuación:

- Especificar el tiempo de finalización del análisis.

- Controlar el tamaño del paso para cada uno de los tiempos.

- Crear múltiples pasos cuando sea necesario.

Los tiempos de análisis fueron determinados por las tasas de enfriamiento proporcionadas por las mediciones experimentales de temperatura realizadas con las termocuplas.

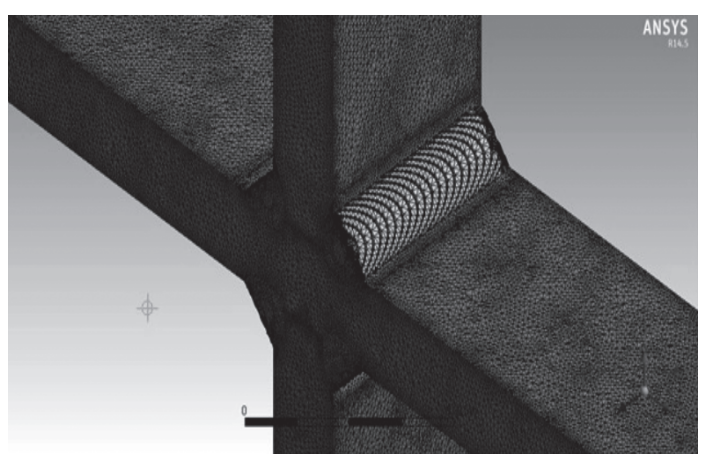

Figura 5. Probeta modelada en ANSYS 14.5.
Las consideraciones y restricciones principales empleadas en el modelo son:

- Tanto el material de aporte (E6013) y el material base (ASTM A36) se consideran elástico lineal y las propiedades se definen de acuerdo con certificado de garantía del proveedor y por comprobación mediante ensayos a tracción.

- La temperatura ambiente se considera de $27^{\circ} \mathrm{C}$.

- Se define el perfil de temperatura inicial de las probetas; las temperaturas obtenidas producto del ensayo termográfico para los diferentes catetos objeto de estudio se extrapolan punto a punto (utilizando el software SmartView) y se utilizan como insumo para las condiciones iniciales de la simulación.

El procedimiento de modelamiento empleado en el software ANSYS 14.5 se indica a continuación:

1. En el módulo Steady-State Thermal se ingresa el perfil de temperatura inicial.

2. El análisis de enfriamiento se realiza en Transient Thermal para cada uno de los medios de enfriamiento y catetos de soldadura objeto de estudio.

3. Para la obtención de los esfuerzos residuales las cargas a aplicar en este análisis serán importadas del análisis de enfriamiento; por ello esta configuración se hace en Imported Load para cada instante de tiempo. Para esto se forma una pareja de datos, donde los tiempos finales de Transient Thermal son entrelazados con los tiempos finales de Transient Structural.

\section{RESULTADOS}

\section{Resultados del modelo numérico}

En la Figura 6 se muestra el resultado del perfil de temperatura inicial para la probeta con cateto de soldadura de $3 \mathrm{~mm}$, este perfil es utilizado como un dato de condición inicial en el software ANSYS 14.5.

Los perfiles de temperatura fueron obtenidos para las probetas con las dimensiones de cateto objeto de estudio. Se observa que los sectores que tienen mayor concentración de temperatura se encuentran en las cercanías de los cordones soldados, disminuyendo en función de la distancia hasta llegar a una temperatura cercana al ambiente. Con la información del perfil de temperatura se procede a realizar el modelamiento 


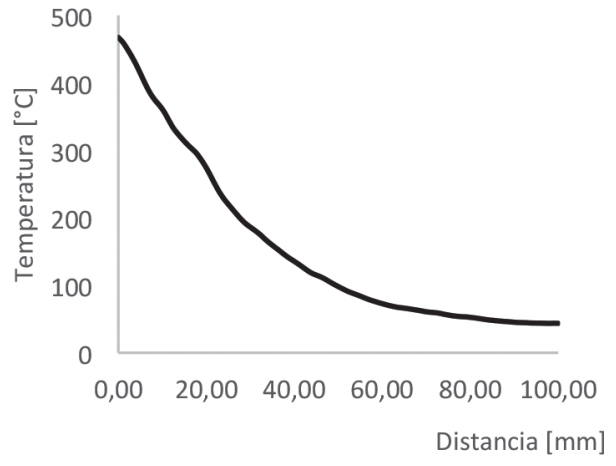

Figura 6. Perfil de temperatura inicial para la probeta con cateto de soldadura de $3 \mathrm{~mm}$.

del enfriamiento para los distintos medios de enfriamiento y los tamaños de cateto empleados en la investigación. En la Figura 7 se observa una de las curvas de enfriamiento obtenidas mediante el modelamiento, estas se confrontan con los ensayos de validación de los modelos.

Como resultado del modelamiento se obtiene que la velocidad de enfriamiento es mayor para las probetas enfriadas en agua y menor para las enfriadas en aceite, de igual forma los catetos de soldadura mayores disminuyen proporcionalmente la velocidad de enfriamiento. Posteriormente se realiza la simulación de los esfuerzos residuales producto del enfriamiento. En la Figura 8 ( $a, b$ y c) se muestra la distribución de los esfuerzos residuales obtenidos por modelamiento para la probeta con cateto de soldadura de $3 \mathrm{~mm}, 4 \mathrm{~mm}$, $5 \mathrm{~mm}$, respectivamente.

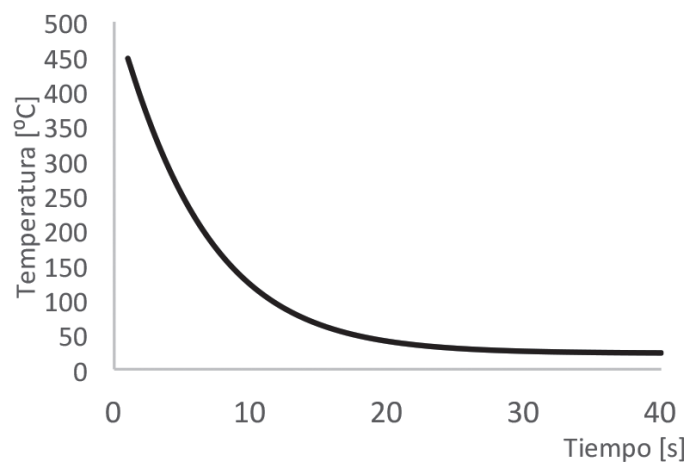

Figura 7. Curva de enfriamiento obtenida del modelo teórico para medio de enfriamiento agua y probeta con cateto de soldadura de $3 \mathrm{~mm}$.
Como resultado se obtiene que una mayor dimensión de cateto de soldadura provoca mayores magnitudes de los esfuerzos residuales; por otro lado, los medios con mayores tasas de enfriamiento generan regiones de influencia mayores.

\section{Experimentación y validación de los modelos}

Se realizaron los ensayos de enfriamiento a nueve probetas con la configuración requerida, a las cuales se les instala termocuplas tipo K para la obtención de los datos de enfriamiento. En la Figura 9 (a) aparece el montaje empleado para una probeta al momento de la medición de la tasa de enfriamiento; mientras que en la Figura 9 (b) se muestra una de las curvas de enfriamiento obtenidas. Se obtiene como resultado una muy buena correspondencia entre las curvas

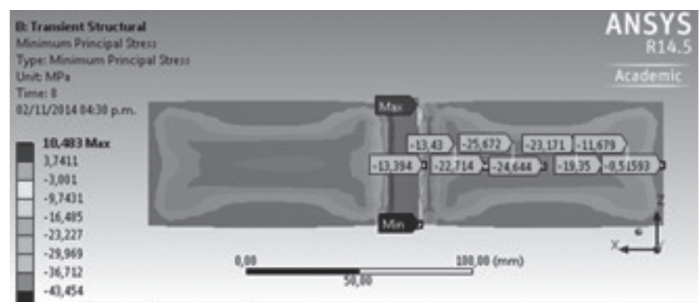

(a) Probeta con cateto de soldadura de $3 \mathrm{~mm}$, medio de enfriamiento aceite.

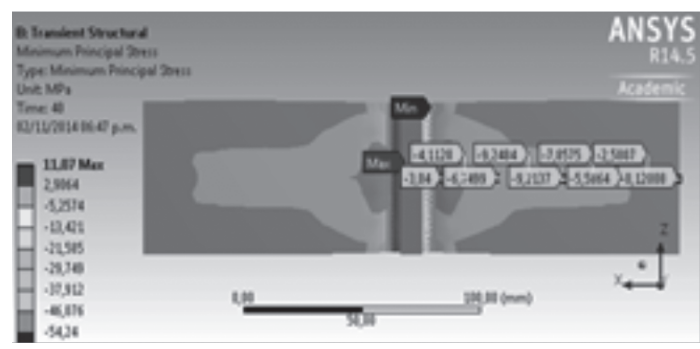

(b) Probeta con cateto de soldadura de $4 \mathrm{~mm}$, medio de enfriamiento aire.

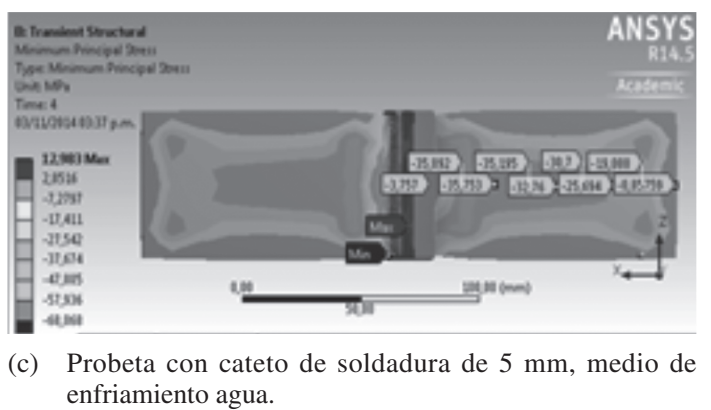

Figura 8. Distribución de los esfuerzos residuales de acuerdo con los resultados del modelo numérico. 
(a)



(b)

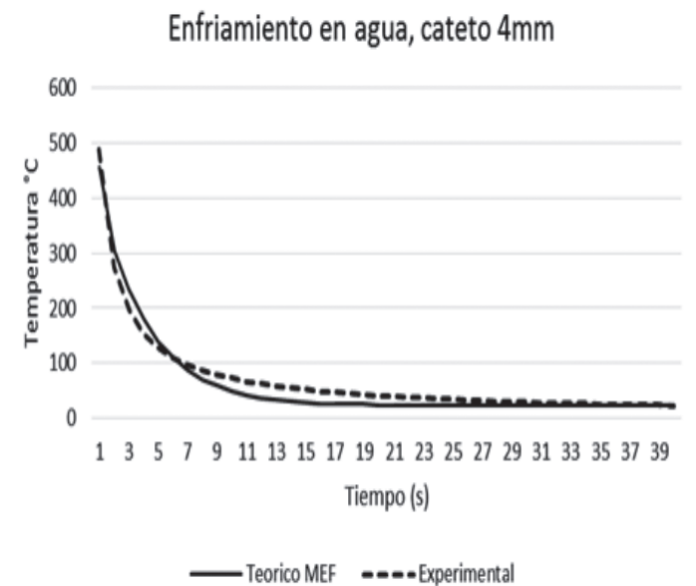

Figura 9. (a) Probeta en proceso de soldadura con las termocuplas instaladas; (b) Curva de enfriamiento experimental y teórica (MEF) para la probeta con medio de enfriamiento agua y cateto de soldadura de $4 \mathrm{~mm}$.

de enfriamiento producto de las simulaciones y las registradas mediante los ensayos experimentales.

En la Tabla 4 aparecen las tasas de enfriamiento obtenidas para cada tratamiento establecido; las cuales han sido calculadas para un intervalo de tiempo transcurrido desde la temperatura inicial hasta una temperatura de $200^{\circ} \mathrm{C}$.

La magnitud de los esfuerzos residuales obtenida de las simulaciones realizadas se muestra en la Tabla 5, para el caso de enfriamiento de las probetas tratadas y para las tres dimensiones de cateto analizadas.

Como técnica experimental para medir los esfuerzos residuales se empleó DFX, utilizando un equipo
Tabla 4. (a). Tasa de enfriamiento teórico para cada tratamiento empleado $\left({ }^{\circ} \mathrm{C} / \mathrm{s}\right)$.

\begin{tabular}{|c|c|c|c|}
\hline \multirow{2}{*}{$\begin{array}{c}\text { Medio de } \\
\text { enfriamiento }\end{array}$} & \multicolumn{3}{|c|}{$\begin{array}{c}\text { Dimensión del cateto de } \\
\text { soldadura (mm) }\end{array}$} \\
\cline { 2 - 4 } & $\mathbf{3}$ & $\mathbf{4}$ & $\mathbf{5}$ \\
\hline Agua & $-87,34$ & $-107,81$ & $-112,02$ \\
\hline Aceite & $-62,42$ & $-66,70$ & $-70,90$ \\
\hline Aire & $-8,426$ & $-8,83$ & $-8,97$ \\
\hline
\end{tabular}

(b).Tasa de enfriamiento experimental para cada tratamiento empleado $\left({ }^{\circ} \mathrm{C} / \mathrm{s}\right)$.

\begin{tabular}{|c|c|c|c|}
\hline \multirow{2}{*}{$\begin{array}{c}\text { Medio de } \\
\text { enfriamiento }\end{array}$} & \multicolumn{3}{|c|}{$\begin{array}{c}\text { Dimensión del cateto de } \\
\text { soldadura (mm) }\end{array}$} \\
\cline { 2 - 4 } & $\mathbf{3}$ & $\mathbf{4}$ & $\mathbf{5}$ \\
\hline Agua & $-112,28$ & $-116,58$ & $-131,36$ \\
\hline Aceite & $-42,07$ & $-71,70$ & $-76,16$ \\
\hline Aire & $-9,15$ & $-13,58$ & $-14,97$ \\
\hline
\end{tabular}

Tabla 5. Esfuerzos residuales máximos (MPa).

\begin{tabular}{|c|c|c|c|}
\hline \multirow{2}{*}{$\begin{array}{c}\text { Medio de } \\
\text { enfriamiento }\end{array}$} & \multicolumn{3}{|c|}{$\begin{array}{c}\text { Dimensión del cateto de } \\
\text { soldadura (mm) }\end{array}$} \\
\cline { 2 - 4 } & $\mathbf{3}$ & $\mathbf{4}$ & $\mathbf{5}$ \\
\hline Aire & $-87,97$ & $-91,49$ & $-97,10$ \\
\hline Aceite & $-90,07$ & $-98,23$ & $-110,60$ \\
\hline Agua & $-101,30$ & $-117,01$ & $-119,12$ \\
\hline
\end{tabular}

Panalytical X'Pert PRO MPD, los valores de esfuerzos residuales obtenidos por medio del modelo numérico resultaron similares a los medidos por la técnica experimental, quedando dentro del intervalo de dispersión.

Varios trabajos han sido publicados con resultados sobre el ciclo térmico y la determinación de los esfuerzos residuales que se generan en uniones soldadas [18-23]. Producto de la gran variedad principalmente de los procesos de soldadura, materiales empleados y geometrías de las piezas a soldar, es difícil encontrar un trabajo de investigación con un objeto de estudio igual al empleado aquí. No obstante sí es posible establecer ciertas comparaciones con trabajos de otros autores. Por ejemplo, Chaowen y Yong [24] en su estudio teórico experimental acerca del proceso de soldadura en servicio obtienen curvas de enfriamiento similares 
a las obtenidas en este trabajo para un acero X70, para condiciones de contacto del material base con agua. Las tasas de enfriamiento resultaron ser similares, ocurriendo un rápido enfriamiento de la unión soldada. Sin embargo, los valores de esfuerzos residuales reportados para una ubicación similar a la estudiada se encontraron en el rango de 300-350 MPa, bastante diferentes a los medidos y modelados en la presente investigación. Por otro lado, en [25] se presenta una investigación teórico experimental respecto del ciclo térmico para la soldadura de una unión tipo T en acero AISI 304 para distintos espesores de placa $(5,6$ y $8 \mathrm{~mm})$. Se emplean en esta investigación modelos de elementos finitos en $2 \mathrm{D}$ y $3 \mathrm{D}$, respectivamente; este último es el que arroja los mejores resultados, logrando predecir el comportamiento termomecánico con una exactitud aceptable. Las tasas de enfriamiento que se miden experimentalmente y que resultan del modelamiento numérico por estos autores llegan a ser en promedio $22 \%$ de las mostradas en la presente investigación, para igual espesor de placa y condición de enfriamiento al aire, respectivamente. Lo anterior se debe con seguridad a la menor conductividad del acero inoxidable respecto de un acero estructural de bajo contenido de carbono. Los esfuerzos residuales no fueron analizados en este último trabajo.

\section{CONCLUSIONES}

Se formuló un modelo basado en el método de los elementos finitos para simular el proceso de enfriamiento postsoldadura y el surgimiento del campo de esfuerzos residuales. Este modelo se definió de tipo no lineal transitorio y mostró convergencia para un enmallado de aproximadamente 1,5 millones de nodos. Como aportes de este trabajo de modelamiento se mencionan haber emulado las características superficiales del cordón de soldadura real en el modelo geométrico y el emplear un campo de temperatura inicial definido de acuerdo con mediciones experimentales. También se planteó un diseño experimental para el estudio empírico del proceso de enfriamiento postsoldadura de probetas cruciformes. Se emplearon dos métodos experimentales para la determinación de la temperatura en las probetas soldadas cruciformes: termográfico y mediante colocación directa de termocuplas. Lo anterior permitió encontrar la relación entre el campo de temperatura inicial y su evolución en el tiempo, con relación a los factores experimentales, dimensión del cateto de soldadura y medio de enfriamiento, respectivamente. Adicionalmente se determinaron los esfuerzos residuales inducidos en el proceso de soldadura mediante la técnica de difracción por rayos-X. Se encontró buena correspondencia entre el modelo teórico basado en elementos finitos y los resultados experimentales. En términos generales el modelo numérico de enfriamiento posee un buen grado de aproximación a la curva de enfriamiento medida experimentalmente. La máxima desviación promedio calculada entre el modelo experimental y el teórico fue de $41,5^{\circ} \mathrm{C}$; mientras que la máxima desviación entre los dos modelos fue de $62,7^{\circ} \mathrm{C}$. Ambos valores ocurren para el cordón de soldadura de $3 \mathrm{~mm}$ empleando como medio de enfriamiento agua. En general, considerando la desviación promedio global de todas las simulaciones se logra una variación entre los modelos teórico y físico de $19,5 \%$; lo anterior pone de manifiesto muy buena correspondencia entre los valores teóricos y experimentales, se puede concluir que catetos de soldadura de mayor dimensión generan zonas de calentamiento más extensas y mayores valores de temperatura; por ejemplo, para el cateto de $5 \mathrm{~mm}$ se genera una temperatura pico $5 \%$ mayor que para el cateto de soldadura de $3 \mathrm{~mm}$, para la zona inmediatamente continua a la deposición del cordón. Este comportamiento puede explicarse producto a la mayor cantidad de calor aportado tanto a la junta soldada como a las placas, cuando el cateto de soldadura es mayor. Además, se puede apreciar que los medios de enfriamiento más intensos provocan tasas de enfriamiento postsoldadura de mayor magnitud, lo que trae como consecuencia zonas de influencia de los esfuerzos residuales más extensas y mayores valores de los esfuerzos residuales; por ejemplo, los esfuerzos residuales máximos medidos para las probetas enfriadas en agua son en promedio $20 \%$ mayores que los registrados en las probetas que fueron enfriadas al aire. Este comportamiento se debe a que los mayores gradientes de temperatura y los cambios de fases en mayor extensión volumétrica, que generan los medios de enfriamiento más intensos, producen desequilibrio mecánico a escala microestructural que son más extensos; lo anterior se traduce en esfuerzos internos de mayor magnitud una vez que se alcanza el equilibrio térmico en las probetas. Como dirección de trabajo futura se plantea el 
estudio de la microestructura resultante del proceso de soldadura para la zona de aporte y la ZAC, con el fin de correlacionar los campos de temperatura postsoldadura y los esfuerzos residuales con la composición microestructural para las distintas localizaciones.

\section{AGRADECIMIENTOS}

Los autores desean agradecer el soporte brindado para llevar a cabo esta investigación a la Dirección de Investigaciones de la Universidad de Ibagué (Proyecto de Investigación 12-256-COL0007284), Colombia; WEST ARCO, Bogotá, Colombia, y a la Dirección Nacional de Investigación de la Sede Bogotá - Universidad Nacional de Colombia (Proyecto de Investigación DIB 8008044).

\section{REFERENCIAS}

[1] F. Ramos Morales, A. Duffus Scott, M. Rodríguez Pérez, E. Díaz Cedré, J. Pozo Morejón y M. Oria. "Modelo de elementos finitos para determinar el campo de temperatura en una costura de filete". Ingeniería, Revista Académica de la FIUADY. Vol. 11, Issue 3, pp. 33-42. 2007.

[2] M. Attarha and I. Sattari-far. "Study on welding temperature distribution in thin welded plates through experimental measurements and finite element simulation". Journal of Materials Processing Technology. Vol. 211, Issue 4, pp. 688-694. 2011.

[3] H. Rodríguez. "Metalurgia de la soldadura". Ciudad de La Habana. Editorial Pueblo y Educación. Cuba. 1988.

[4] L. Hwa Teng and W. Jia Lin. "The effects of peak temperature and cooling rate on the susceptibility to intergranular corrosion of alloy 690 by laser beam and gas tungsten arc welding". Corrosion Science. Vol. 51, Issue 3, pp. 439-445. 2009.

[5] L. Fratini, G. Buffa and R. Shivpuri. "Mechanical and metallurgical effects of in process cooling during friction stir welding of AA7075-T6 butt joints". Acta Materialia. Vol. 58, Issue 6, pp. 2056-2067. 2010.

[6] H. Teng Lee, C. Te Chen and J. Lin Wu. "Numerical and experimental investigation into effect of temperature field on sensitization of Alloy 690 butt welds fabricated by gas tungsten arc welding and laser beam welding". Journal of Materials Processing Technology. Vol. 210, Issue 12, pp. 1636-1645. 2010.

[7] S. Yaowu, C. Dong, L. Yongping and L. Xiaoyan. "HAZ microstructure simulation in welding of a ultra fine grain steel". School of Materials Science and Engineering Computational. Materials Science. Vol. 31, Issues 3-4, pp. 379-388. 2004.

[8] W. Perret, C. Schwenk and C. Rethmeier. "Comparison of analytical and numerical welding temperature field calculation". Computational Materials Science. Vol. 47, Issue 4, pp. 1005-1015. 2010.

[9] M. Wahab, M. Painter and M. Davies. "The prediction of the temperature distribution and weld pool geometry in the gas metal arc welding process". Journal of Materials Processing Technology. Vol. 77, Issues 1-3, pp. 233-239. 1998.

[10] LV. Shixiong, S. Jianling, W. Haitao and Y. Shiqin. "Temperature field and flow field during tungsten inert gas bead welding of copper alloy onto steel". Materials Science and Engineering. Vol. 499, Issue 1-2, pp. 347351. 2009.

[11] I. Mejía, C. Maldonado, A. Bedolla y Ch. J. García. "Determinación de tipo, magnitud y dirección de esfuerzos residuales generados en la soldadura de acero AISI H-13 mediante el método de hole drilling. CONAMET/ SAM. Santiago, Chile. 2006.

[12] I. Mejía, C. Maldonado, A. Bedolla y Ch. J. García. "Esfuerzos residuales generados en la soldadura de placas de acero inoxidable AISI 304 mediante el proceso SMAW". CONAMET/SAM. Santiago, Chile. 2006.

[13] W. Zinn, B. Scholtes. "Handbook of Residual Stress and Deformation Steel". edited by G. Totten, M. Howes and T. Inoue. American Society of Materials International, pp. 391396. 2001.

[14] M. Monsalve, M. López y F. Vargas. "Técnicas utilizadas para la medición de esfuerzos residuales en películas delgadas depositadas por PVD”. Scientia et Technica. Vol. XIII, $\mathrm{N}^{\circ}$ 36, pp. 765-770. 2007.

[15] A.J. Aguirre. "Influencia de las tensiones residuales de mecanizado en las propiedades mecánicas de Y-TZ”. Tesis de grado. Universidad Politécnica de Cataluña. España. 2004. 
[16] AWS. "Design Handbook for calculating fillet weld sizes". American Welding Society. Miami, Florida. 1997.

[17] N. Arzola and O. Araque. "Chord profile influence on the fatigue failure of a t-butt weld joint". Ingeniare. Revista chilena de ingeniería. Vol. $22 \mathrm{~N}^{\circ}$ 2, pp. 196-204. 2014.

[18] C. Heinze, C. Schwenk and M. Rethmeier. "Numerical calculation of residual stress development of multi-pass gas metal arc welding”. J Construct Steel Res. Vol. 72, pp. 12-19. 2012.

[19] Z. Barsouma and A. Lundbäck. "Simplified FE welding simulation of fillet welds-3D effects on the formation residual stresses". J Eng Fail Anal. Vol. 16, pp. 2281-2289. 2009.

[20] MC. Smith, PJ. Bouchard, M. Turski, L. Edwards and RJ. Dennis. "Accurate prediction of residual stress in stainless steel welds". J Comput Mater Sci. Vol. 54, pp. 312-328. 2012.

[21] Z. Barsoum and A. Lundback. "Simplified FE welding simulation of fillet welds - 3D effects on the formation residual stresses". J Eng Fail Anal. Vol. 16, Issue 7, pp. 22812289. 2009.

[22] TL. Teng, CP. Fung, PH. Chang and WC. Yang. "Analysis of residual stresses and distortions in T-joint fillet welds". J Pressure Ves Pip. Vol. 78, Issue 8, pp. 523-38. 2001.

[23] D. Deng. "Influence of deposition sequence on welding residual stress and deformation in an austenitic stainless steel J-groove welded joint". Mater Des. Vol. 49, pp. 10221033. 2013.

[24] L. Chaowen and W. Yong. "Three-dimensional finite element analysis of temperature and stress distributions for in-service welding process". Materials and Design. Vol. 52, pp. 1052-1057. 2013.

[25] F. Vakili-Tahami and A. Ziaei-Asl. "Numerical and experimental investigation of T-shape fillet welding of AISI 304 stainless steel plates". Materials and Design. Vol. 47, pp. 615-623. 2013. 